\title{
Analytic cohomology in Fréchet spaces
}

\author{
LÁSZLÓ LEMPERT
}

\begin{abstract}
We consider a certain class of Fréchet spaces that includes the space $s$ of rapidly decreasing sequences, and prove that if $\Omega$ is a pseudoconvex open subset of a space in this class then $H^{q}(\Omega, \mathcal{O})=$ $0, q \geq 1$.
\end{abstract}

\section{Introduction.}

The computation of sheaf cohomology groups has been a central problem of complex analysis and geometry for over fifty years now. It appears that in an infinite dimensional setting sheaf cohomologies were first investigated by Douady in [Do]. In this paper we address the question whether the cohomology groups $H^{q}(\Omega, \mathcal{O})$ vanish when $q \geq 1$ and $\Omega$ is a pseudoconvex open set in a complex Fréchet space $X$. Here $\mathcal{O}$ denotes the sheaf of germs of holomorphic functions in $X$ and $\Omega$ is called pseudoconvex if $\Omega \cap Z$ is pseudoconvex for all finite dimensional subspaces $Z \subset X$; in particular $X$ itself is pseudoconvex.

By now it has become clear that coholomogy vanishing is sensitive to the geometry of the space $X$ in question. After the first examples of nonvanishing by Dineen, Meise and Vogt formulated a necessary condition for $H^{1}(X, \mathcal{O})=$ 0 to hold, see [D1,MV]. This condition, the existence of a dominant norm, or (DN), is met by all Banach spaces and also by the space $s$ of rapidly decreasing sequences (and its subspaces). ${ }^{2}$ On the other hand we proved in [L1] that $H^{q}(\Omega, \mathcal{O})=0$ if $\Omega$ is pseudoconvex in a Banach space $X$ with unconditional basis and $q \geq 1$; for example $X$ could be the space $l^{p}, 1 \leq p<$ $\infty$, or $L^{p}[0,1], 1<p<\infty$. Here we shall show that it is possible to prove cohomology vanishing in nonnormable Fréchet spaces as well, in particular in $s$. The space $s$ deserves special attention among Fréchet spaces as function spaces that frequently occur in geometry are isomorphic to it; it also has certain universality properties.

\footnotetext{
${ }^{1}$ Research partially supported by an NSF grant.

${ }^{2}$ Technically, [MV] deals with Dolbeault rather than sheaf cohomology groups, but the reasoning there also shows that $H^{1}(X, \mathcal{O})=0$ implies $(\mathrm{DN})$.
} 
We shall consider sequence spaces defined as follows. Fix a function $p: \mathbb{R} \times \mathbb{N} \rightarrow(0, \infty)$ such that $\log p(\cdot, n)$ is convex for all $n \in \mathbb{N}$. If $x: \mathbb{N} \rightarrow \mathbb{C}$ and $\theta \in \mathbb{R}$, set

$$
\begin{aligned}
& \|x\|_{\theta}=\sum_{n} p(\theta, n)|x(n)|, \quad \text { and } \\
& X=\left\{x: \mathbb{N} \rightarrow \mathbb{C} \mid\|x\|_{\theta}<\infty \text { for all } \theta \in \mathbb{R}\right\} .
\end{aligned}
$$

The norms \|\|$_{\theta}, \theta \in \mathbb{R}$, endow $X$ with the structure of a complete locally convex topological vector space. Convexity implies that the norms \|\|$_{\theta}, \theta \in \mathbb{Z}$, would induce the same topology, so that $X$ is a Fréchet space. For instance, if $p(\theta, n)=n^{\theta}$ then $X \approx s$.

Theorem 1.1. Let $X$ be as above, $\Omega \subset X$ open and pseudoconvex. Let $F$ be an arbitrary Fréchet space and $\mathcal{F}$ the sheaf of germs of holomorphic functions on $X$ with values in $F$. Then

$$
H^{q}(\Omega, \mathcal{F})=0, \quad q \geq 1
$$

A noteworthy aspect of this theorem is the convexity condition imposed on $\log p$. As already discussed in [L2], convexity is a natural assumption in complex analysis; the novel feature is that here it pertains to the topology of $X$.

We are now going to sketch the proof of Theorem 1.1, an extension of the one in [L1] that dealt with Banach spaces. The chief difference between the Banach and Fréchet settings is that when $\Omega$ is a pseudoconvex open subset of a Banach space, the function - log $\operatorname{dist}(\cdot, \partial \Omega)$ is plurisubharmonic; but this is not necessarily so in a Fréchet space such as $X$. Accordingly, the exhaustion procedure of [L1] will have to be replaced by a two step procedure as follows. We shall mostly work with open coverings $\mathfrak{U}$ of $\Omega$ and the corresponding Cech groups $H^{q}(\mathfrak{U}, \mathcal{F})$. First we write $X=\mathbb{C}^{N} \oplus Y$, assume that $\Omega=D \times G$ with $D \subset \mathbb{C}^{N}, G \subset Y$, and that elements of $\mathfrak{U}$ are of form $V \times G, V \subset D$ pseudoconvex. In this case $H^{q}(\mathfrak{U}, \mathcal{F})=0$ follows from the finite dimensional theory. Second we introduce a translation invariant metric in $X$ and with a general pseudoconvex $\Omega$ and $b>0$ we consider the set $\Omega[b]$ of points $x \in \Omega$ at distance $>b$ to $\partial \Omega$. We approximate $\Omega[b]$ by product sets $\Omega_{N}$ as in the first step, and show that vanishing for $\Omega_{N}$ implies $H^{q}(\mathfrak{B}, \mathcal{F}) \mid \Omega[b]=0$ for certain coverings $\mathfrak{B}$ of $\Omega$ by balls. Third, by letting $b \rightarrow 0$ we conclude $H^{q}(\mathfrak{B}, \mathcal{F})=0$. Finally we show this latter implies $H^{q}(\Omega, \mathcal{F})=0$.

The narrow cross section of the proof is steps 2 and 3. Both steps concern continuity of cohomology groups and depend on the approximation theorem 
from [L2]. It is but for that approximation theorem that we had to restrict ourselves to spaces $X$ as in (1.1).

We assume the reader has some familiarity with complex analysis in finite and infinite dimensions and with sheaf theory. Some good references are $[\mathrm{B}, \mathrm{D} 2, \mathrm{H}, \mathrm{Ma}, \mathrm{N}]$.

\section{Background.}

Here we collect notation and background material that can also be found in [L1] or follows directly from [L1]. We shall use standard notation of sheaf theory. If $\mathfrak{U}$ is an open covering of a topological space $T$, we call an ordered $(q+1)$ tuple $U_{0}, \ldots, U_{q} \in \mathfrak{U}$ a $q$-simplex and denote it by $\sigma ;|\sigma|$ stands for $U_{0} \cap \cdots \cap U_{q}(q=0,1, \ldots)$. We shall also define a $(-1)$-simplex $\sigma$; its body $|\sigma|$ is all of $T$. Given a sheaf $\mathcal{S}$ of Abelian groups on $T$, a $q$ cochain $f$ is a collection of $f_{\sigma} \in \mathcal{S}(|\sigma|)$, one for each $q$-simplex $\sigma$, with the understanding that $\mathcal{S}(\emptyset)=(0)$. The group of $q$-cochains is denoted $C^{q}(\mathfrak{U}, \mathcal{S})$; $\delta: C^{q}(\mathfrak{U}, \mathcal{S}) \rightarrow C^{q+1}(\mathfrak{U}, \mathcal{S})$ is the coboundary homomorphism, and $H^{q}(\mathfrak{U}, \mathcal{S})$ are the corresponding cohomology groups.

Suppose that for each $U \in \mathfrak{U}$ we are given an open $U^{\prime} \subset U$, and let $\left\{U^{\prime}\right\}=\mathfrak{U}^{\prime}$. Then we have restriction homomorphisms

$$
C^{q}(\mathfrak{U}, \mathcal{S}) \rightarrow C^{q}\left(\mathfrak{U}^{\prime}, \mathcal{S}\right), \quad H^{q}(\mathfrak{U}, \mathcal{S}) \rightarrow H^{q}\left(\mathfrak{U}^{\prime}, \mathcal{S}\right),
$$

and the image of a cochain resp. cohomology class $f$ will be denoted $f \mid \mathfrak{U}^{\prime}$. In particular, any open $T^{\prime} \subset T$ induces a covering $\mathfrak{U}^{\prime}=\mathfrak{U} \mid T^{\prime}=\left\{U^{\prime}=U \cap T^{\prime}\right.$ : $U \in \mathfrak{U}\}$ of $T^{\prime}$. In this case the homomorphisms (1.1) are induced by the embedding $T^{\prime} \rightarrow T$. We shall simply denote the image $f \mid \mathfrak{U}^{\prime}$ of a cochain resp. cohomology class $f$ by $f \mid T^{\prime}$, and the image of the homomorphisms $(2.1)$ by $C^{q}(\mathfrak{U}, \mathcal{S}) \mid T^{\prime}$ resp. $H^{q}(\mathfrak{U}, \mathcal{S}) \mid T^{\prime}$. We shall also use the corresponding notation $H^{q}(T, \mathcal{S}) \mid T^{\prime}$ for the sheaf cohomology groups.

We shall say that $\mathfrak{U}$ is a Leray covering of order $q$ if for all $1 \leq s<q$ and all $p$-simplices $\sigma \subset \mathfrak{U}, p \geq 0$, the groups $H^{s}(|\sigma|, \mathcal{S})$ vanish. We shall need the following result (see [L1, Proposition 1.1]).

Proposition 2.1. Suppose $\mathfrak{V}$ is a Leray covering of order $q$ for the sheaf $\mathcal{S}$ on $T$.

(a) The natural map

$$
H^{q}(\mathfrak{V}, \mathcal{S}) \rightarrow H^{q}(T, \mathcal{S})
$$


is a monomorphism, and its image consists of those $f \in H^{q}(T, \mathcal{S})$ that satisfy $f \mid V=0$ for all $V \in \mathfrak{V}$.

(b) If $\mathfrak{V}^{\prime}$ is a refinement of $\mathfrak{V}$, then the refinement map

$$
H^{q}(\mathfrak{V}, \mathcal{S}) \rightarrow H^{q}\left(\mathfrak{V}^{\prime}, \mathcal{S}\right)
$$

is a monomorphism.

Now consider two Fréchet spaces $X$ and $F$ (always over $\mathbb{C}$ ), $\mathcal{F}$ the sheaf of germs of $F$ valued holomorphic functions on $X$. These pieces of notation will be used throughout the paper. Let $\omega \subset X$ be open, $\mathfrak{U}$ a covering of $\omega$ by open $U \subset X, \mathfrak{U}^{\prime}$ another covering gotten by selecting an open subset $U^{\prime} \subset U$ for each $U \in \mathfrak{U}$. If $\sigma=\left(U_{0}, \ldots, U_{s}\right) \subset \mathfrak{U}$, let $\sigma^{\prime}=\left(U_{0}^{\prime}, \ldots, U_{s}^{\prime}\right) \subset \mathfrak{U}$.

Lemma 2.2. Suppose open subsets $\omega_{N}, \Omega_{N} \subset \bigcup \mathfrak{U}$ satisfy $\omega_{N} \subset \Omega_{N} \cap \Omega_{N+1}$, $N=1,2, \ldots$ Fix $q=1,2, \ldots$ and assume

(i) each $z \in \omega$ has a neighborhood that is contained in all but finitely many $\omega_{N}$

(ii) with $\sigma \subset \mathfrak{U}$ denoting an arbitrary $(q-2)$-simplex, any $u \in \mathcal{O}\left(|\sigma| \cap \Omega_{N} \cap\right.$ $\left.\Omega_{N+1} ; F\right)$ can be approximated by $v \in \mathcal{O}\left(|\sigma| \cap \Omega_{N+1} ; F\right)$, uniformly on $\left|\sigma^{\prime}\right| \cap \omega_{N}, N=1,2, \ldots$;

and in case $q \geq 2$ also assume

(iii) $H^{q-1}\left(\mathfrak{U} \mid \Omega_{N} \cap \Omega_{N+1}, \mathcal{F}\right)=0, N=1,2, \ldots$.

If now $H^{q}(\mathfrak{U}, \mathcal{F}) \mid \Omega_{N}=0$ for $N=1,2, \ldots$ then the image of $H^{q}(\mathfrak{U}, \mathcal{F})$ in $H^{q}(\omega, \mathcal{F})$ is zero.

This lemma is a slight extension of [L1, Lemma 2.1], where $X$ was assumed to be a Banach space and $\omega_{N}, \Omega_{N} \subset \omega(=\Omega)$. However, the same proof as there applies here, too.

Next we turn to cohomology groups in product domains. Let $Y$ be an arbitrary locally convex space, $G \subset Y$ and $D \subset \mathbb{C}^{N}$ open, $\omega=D \times G$. We shall use the following pieces of notation. If $V \subset D$ is open, set $V(\omega)=$ $V \times G$; if $\mathfrak{V}$ is an open covering of $D$, set $\mathfrak{V}(\omega)=\{V(\omega): V \in \mathfrak{V}\}$; if $\sigma=\left(V_{0}, \ldots, V_{s}\right) \subset \mathfrak{V}$, set $\sigma(\omega)=\left(\left(V_{0}(\omega), \ldots, V_{s}(\omega)\right)\right.$. Suppose in addition to $D$ and its covering $\mathfrak{V}$ we are also given a pseudoconvex $D^{\star} \subset \mathbb{C}^{N}$ in which $D$ is compactly contained; for each $V \in \mathfrak{V}$ we are given a pseudoconvex $V^{\star} \subset D^{\star}$ in which $V$ is compactly contained; and $\mathfrak{V}^{\star}=\left\{V^{\star}: V \in \mathfrak{V}\right\}$ covers $D^{\star}$. Let $\omega^{\star}=D^{\star} \times G$. 
Proposition 2.3. For $q=1,2, \ldots$ the image of $H^{q}\left(\mathfrak{V}^{\star}\left(\omega^{\star}\right), \mathcal{F}\right) \mid \omega$ in $H^{q}(\omega, \mathcal{F})$ is zero.

Proof. Induce the topology of $F$ by a family $\Psi$ of seminorms, and for any cochain $h=\left\{h_{\sigma}\right\} \in C^{s}(\mathfrak{V}, \mathcal{F})$ and $\psi \in \Psi$ define

$$
\psi_{\mathfrak{V}}(h)=\sup _{\sigma} \sup _{|\sigma|} \psi\left(h_{\sigma}\right) \leq \infty .
$$

Let $C_{\infty}^{s}(\mathfrak{V}, \mathcal{F})$ consist of those $h \in C^{s}(\mathfrak{V}, \mathcal{F})$ that satisfy $\psi_{\mathfrak{V}}(h)<\infty$ for all $\psi \in \Psi$, and $Z_{\infty}^{s}(\mathfrak{V}, \mathcal{F})=\left\{h \in C_{\infty}^{s}(\mathfrak{V}, \mathcal{F}): \delta h=0\right\}$. The seminorms $\psi_{\mathfrak{V}}$ endow both spaces with the structure of a Fréchet space. Define $\psi_{\mathfrak{V}^{\star}}$ etc. analogously. By [L1, Proposition 3.2] there is a continuous linear operator

$$
S: Z_{\infty}^{q}\left(\mathfrak{V}^{\star}, \mathcal{F}\right) \rightarrow C_{\infty}^{q-1}(\mathfrak{V}, \mathcal{F})
$$

such that for any $h \in Z_{\infty}^{q}\left(\mathfrak{V}^{\star}, \mathcal{F}\right)$ we have $\delta S h=h \mid \mathfrak{V}$. We shall reduce the study of cohomology groups of $\omega, \omega^{\star}$ to those of $D, D^{\star}$ by means of embeddings

$$
i_{z}: \mathbb{C}^{N} \ni \zeta \mapsto(\zeta, z) \in \mathbb{C}^{N} \oplus Y, \quad z \in \dot{G} .
$$

To prove our Proposition, let $f \in C^{q}\left(\mathfrak{V}^{\star}\left(\omega^{\star}\right), \mathcal{F}\right)$ be closed. At the price of shrinking the sets $V^{\star} \in \mathfrak{V}^{\star}$ it can be assumed that the components $f_{\sigma^{\star}\left(\omega^{\star}\right)}$ of $f$ extend continuously to the closure of $\left|\sigma^{\star}\left(\omega^{\star}\right)\right|$ in $\omega^{\star}$. In this case we have a holomorphic map

$$
G \ni z \mapsto i_{z}^{*} f \in Z_{\infty}^{q}\left(\mathfrak{V}^{\star}, \mathcal{F}\right),
$$

see [L1, Proposition 4.2], whose simple proof carries over to our setting. Let $g^{z}=S i_{z}^{*} f ;$ then $\delta g^{z}=i_{z}^{*} f \mid \mathfrak{V}$. Since $g^{z} \in C_{\infty}^{q-1}(\mathfrak{V}, \mathcal{F})$ depends holomorphically on $z \in G$ one easily constructs a (unique) $g \in C^{q-1}(\mathfrak{V}(\omega), \mathcal{F})$ such that $i_{z}^{*} g=g^{z}$, as in [L1, Proposition 4.3]. It is immediate that $\delta g=f \mid \mathfrak{V}(\omega)$, so that the image of $f \mid \mathfrak{V}(\omega)$ in $H^{q}(\mathfrak{V}(\omega), \mathcal{F})$, hence also in $H^{q}(\omega, \mathcal{F})$, is indeed zero.

\section{Exhaustions.}

In this section, after some preparation, we shall approximate general open sets $\Omega$ by simpler ones, and describe properties of the approximating sets. From now on we assume that $X, F$, and $\mathcal{F}$ are as in Theorem 1.1. All 
through the section we shall also assume that the weight $p(\theta, n)$ is even in $\theta$. In particular, it increases with $\theta \geq 0$.

Recall that a pseudonorm on $X$ is a function \|\|$: X \rightarrow[0, \infty)$ such that $\|x\|=0$ if and only if $x=0 ;\|\lambda x\| \leq\|x\|$ if $\lambda \in \mathbb{C},|\lambda| \leq 1$; and $\|x+y\| \leq\|x\|+\|y\|$.

\section{Proposition 3.1. The formula}

$$
\|x\|=\inf \left\{\theta>0:\|x\|_{1 / \theta} \leq \theta\right\}
$$

defines a pseudonorm that induces the topology of $X$, and $\|x\|<r$ is equivalent to $\|x\|_{1 / r}<r$. Also, $\log \|\|$ is plurisubharmonic.

Proof. All but the last statement is already in [L2, Proposition 5.1]. To prove plurisubharmonicity, first consider a convex function $F: \mathbb{R}^{N} \times \mathbb{R} \rightarrow \mathbb{R}$ such that as $\eta$ increases from $-\infty$ to $\infty$, so does $F(\xi, \eta)$, for all $\xi \in \mathbb{R}^{N}$; and observe that the unique solution $\eta=G(\xi)$ of the equation $F(\xi, \eta)=0$ is a concave function of $\xi \in \mathbb{R}^{N}$. Indeed, for fixed $\bar{\xi}$ and $\bar{\eta}=G(\bar{\xi})$ there are linear forms $L: \mathbb{R}^{N} \rightarrow \mathbb{R}, l: \mathbb{R} \rightarrow \mathbb{R}$ such that for all $\xi, \eta$

$$
F(\xi, \eta) \geq F(\bar{\xi}, \bar{\eta})+L(\xi-\bar{\xi})+l(\eta-\bar{\eta}) ;
$$

it follows that $l$ is strictly increasing. Writing $\eta=G(\xi)$ we obtain $0 \geq$ $L(\xi-\bar{\xi})+l(\eta-\bar{\eta})$, or

$$
G(\xi) \leq G(\bar{\xi})-l^{-1} L(\xi-\bar{\xi}) .
$$

Thus the graph of $G$ is below a supporting hyperplane at $(\bar{\xi}, \bar{\eta})$, and so $G$ is concave.

We shall now prove $\log \|\|$ is plurisubharmonic on the subspaces $X_{N}=$ $\{x \in X: x(n)=0$ if $n>N\}, N=1,2, \ldots$ Since it is continuous on $X$, and $\bigcup_{N} X_{N}$ is dense in $X$, it will follow that $\log \|\|$ is plurisubharmonic on $X$ as well.

Continuity of $\|x\|_{1 / \theta}$ implies that for $x \in X_{N} \backslash\{0\}, \theta=\|x\|$ is the unique solution of the equation $\|x\|_{1 / \theta}=\theta$. Thus

$$
\begin{aligned}
& \|x\|^{-1} \sum_{n} p\left(\|x\|^{-1}, n\right)|x(n)|=1, \quad \text { or, taking logarithm } \\
& F(\log |x(1)|, \ldots, \log |x(N)|,-\log \|x\|)=0,
\end{aligned}
$$

where $F(\xi, \eta)=\eta+\log \sum_{n=1}^{N} p\left(e^{\eta}, n\right) e^{\xi_{n}}$, and we assume temporarily that $x(n) \neq 0,1 \leq n \leq N$. Now the sum of logarithmically convex functions is also logarithmically convex. For functions of one variable this is 
proved e.g. in [L2, Lemma 2.1], and the general case follows by restricting to lines. We conclude that $F$ is convex. By our initial observation $-\log \|x\|=G(\log |x(1)|, \ldots, \log |x(N)|)$ with $G$ concave, hence $-\log \|x\|$ is plurisuperharmonic and $\log \|x\|$ plurisubharmonic on the part of $X_{N}$ where $x(n) \neq 0,1 \leq n \leq N$. Since $\log \|\|$ is continuous on $X_{N}$, with values in $[-\infty, \infty)$, we see it is plurisubharmonic on $X_{N}$ as well.

We shall write $B(x, r)=\{y \in X:\|x-y\|<r\}$, and $\operatorname{dist}(x, A)$ for the distance between $x \in X$ and $A \subset X$, measured in \|\| . For $N \in \mathbb{N}$, let $\pi_{N}: X \rightarrow X$ denote the projection given by $\left(\pi_{N} x\right)(n)=x(n)$ resp. 0 if $n \leq N$ resp. $n>N$. Put also $\pi_{0} x=0, \pi_{\infty} x=x$. (1.1) implies

$$
\left\|\pi_{N} x-\pi_{M} x\right\|_{\theta} \leq\left\|\pi_{n} x-\pi_{m} x\right\|_{\theta}, x \in X, 0 \leq n \leq N \leq M \leq m \leq \infty
$$

for all $\theta \in \mathbb{R}$, hence by (3.1)

$$
\left\|\pi_{N} x-\pi_{M} x\right\| \leq\left\|\pi_{n} x-\pi_{m} x\right\|, \quad x \in X, \quad 0 \leq n \leq N \leq M \leq m \leq \infty .
$$

Let $Y=\operatorname{Ker} \pi_{N}, D$ a complex manifold (or a subset of such) and $R$ : $D \rightarrow(0, \infty]$ continuous. Sets of form

$$
\{(\zeta, z) \in D \times Y:\|z\|<R(\zeta) \quad(\text { resp. } \leq R(\zeta))\}
$$

will be called sets of type (B), with base $D$. Such sets have the following Runge approximation property.

Suppose $D$ is a (finite dimensional) Stein manifold, $D^{\prime} \subset D$ open, $L \subset D^{\prime}$ compact. Given continuous functions $r, R: D^{\prime} \rightarrow(0, \infty]$, set

$$
\Omega^{\prime}=\left\{(\zeta, z) \in D^{\prime} \times Y:\|z\|<R(\zeta)\right\}, \quad A=\{(\zeta, z) \in L \times Y:\|z\| \leq r(\zeta)\} .
$$

Proposition 3.2. If $L$ is $\mathcal{O}(D)$ convex and $r<R$, any $u \in \mathcal{O}\left(\Omega^{\prime} ; F\right)$ can be approximated by $v \in \mathcal{O}(D \times Y ; F)$, uniformly on $A$.

Proof. If $Y$ is replaced by $X$ in (3.3) and in the proposition, [L2,Theorem 5.2] shows that the claim follows from the assumptions on $X$. Since $Y$ with the seminorms \|\|$_{\theta}$ (is isomorphic to a space that) satisfies the same assumptions as $X$, the proposition holds.

Our goal here is to exhaust a general open $\Omega \subset X$ by sets of type (B), and prove approximation results for these sets, Propositions 3.5, 3.7, 3.8. These propositions will be used in the next section, where we shall relate the cohomology groups of $\Omega$ to those of the exhausting sets. Because of the two step exhaustion procedure mentioned in the Introduction, we shall work 
with two families of exhausting sets that will correspond to two choices of the function $d$ below.

If $0<a<1, N=1,2, \ldots$, put

$$
\Omega[a]=\{x \in \Omega: \operatorname{dist}(x, \partial \Omega)>a,\|x\|<1 / a\} .
$$

Let furthermore

$$
d_{0}(x)=\min \{1, \operatorname{dist}(x, \partial \Omega)\}, \quad x \in \Omega,
$$

and with $d: \Omega \rightarrow(0,1]$ either $d_{0}$ or the function identically 1 , and $0<\alpha<1$, put

$$
\Omega_{N}(a, \alpha)=\left\{x \in X: \pi_{N} x \in \Omega[a],\left\|x-\pi_{N} x\right\|<\alpha d\left(\pi_{N} x\right)\right\} .
$$

We shall write $\bar{\Omega}[a], \bar{\Omega}_{N}(a, \alpha)$ for the sets defined as above but the strict inequalities replaced by $\leq$, and $\Omega[a]$ by $\bar{\Omega}[a]$ in the case of $\bar{\Omega}_{N}(a, \alpha)$.

When $d=d_{0}$ we let $a, \alpha$ take arbitrary values in $(0,1)$, but when $d \equiv 1$, we shall require $\alpha<a$. In all cases we have then

$$
\alpha d(y)<\operatorname{dist}(y, \partial \Omega), \quad y \in \bar{\Omega}[a]
$$

hence $\Omega_{N}(a, \alpha) \subset \Omega$. For both choices of $d, d(x)-d(y) \leq\|x-y\|$. This implies $d(x) / 2 \leq d(y) \leq 2 d(x)$, provided $\|x-y\| \leq d(x) / 2$, a fact we shall repeatedly use.

With $e_{M} \in X$ the characteristic function of $\{M\}$ we also introduce the auxiliary function

$$
\begin{gathered}
d^{N+1}(x)=\inf \left\{|u|: u \in \mathbb{C}, x+u e_{N+1} \notin \Omega\right\}, \quad \text { and the sets } \\
L_{N}[a]=\left\{x=y+t e_{N+1} \in \bar{\Omega}[a]: y \in \pi_{N} X, t \in \mathbb{C},|t| \leq d^{N+1}(x) / 2\right\} .
\end{gathered}
$$

By [N, Définition 2.1.3 and Lemme 2.1.5] $-\log d^{N+1}$ is plurisubharmonic in $\Omega$, provided $\Omega$ is pseudoconvex. Write $\operatorname{psh}(\Omega)$ for the family of plurisubharmonic functions on $\Omega$, and say that a closed $C \subset \Omega$ is $\operatorname{psh}(\Omega)$ convex if for every $y \in \Omega \backslash C$ there is a $v \in \operatorname{psh}(\Omega) \operatorname{such}$ that $v(y)>\sup _{C} v$.

Proposition 3.3. Suppose $\Omega$ is pseudoconvex. Then $\bar{\Omega}[a], L_{N}[a]$ are $p \operatorname{sh}(\Omega)$ convex, $\Omega[a]$ is pseudoconvex, and so is $\Omega_{N}(a, \alpha)$ provided $d \equiv 1$ and $\alpha<a$.

Proof. Consider $\bar{\Omega}[a]$. If $y \in \Omega \backslash \bar{\Omega}[a]$ then either $\|y\|>1 / a$ or $\|y-z\|<a$ with some $z \in \partial \Omega$. In the first case $v=\|\| \in \operatorname{psh}(\Omega)$ by Proposition 3.1 
and $v(y)>1 / a \geq \sup _{\bar{\Omega}[a]} v$. In the second case $\|y-z\|_{1 / a}<a$, again by Proposition 3.1. Set $z-y=\xi$ and

$$
v(x)=\sup \{-\log |\lambda|: \lambda \in \mathbb{C}, \quad x+\lambda \xi \notin \Omega\} .
$$

By [N, Définition 2.1.3 and Lemme 2.1.5] $v \in \operatorname{psh}(\Omega)$, and $v(y) \geq 0>$ $\log \left(\|\xi\|_{1 / a} / a\right) \geq \sup _{\bar{\Omega}[a]} v$. We conclude $\bar{\Omega}[a]$ is $\operatorname{psh}(\Omega)$ convex.

In particular this means $\bar{\Omega}[a] \cap Z$ is compact and $\operatorname{psh}(\Omega \cap Z)$ convex for finite dimensional subspaces $Z \subset X$. Since $\Omega[a]=\bigcup_{b>a} \bar{\Omega}[b]$, it follows that $\Omega[a] \cap Z$, hence $\Omega[a]$ is pseudoconvex. The rest of the proposition is proved similarly.

Proposition 3.4. Let $d=d_{0}$ or 1 , and in the latter case assume $\alpha<a$, $\gamma<c$.

(a) If $N \leq M, 2 \beta \leq \alpha$, and $2 a \leq b$ then $\Omega_{N}(b, \beta) \subset \Omega_{M}(a, \alpha)$.

(b) For fixed c, $\gamma$, each $x \in \Omega[2 c]$ has a neighborhood that is contained in all but finitely many $\Omega_{N}(c, \gamma)$.

(c) $\pi_{N} L_{N}[a] \subset \Omega$.

(d) If $2 a \leq c, 4 \gamma \leq \alpha$ then $\pi_{N+1} \Omega_{N}(c, \gamma) \subset L_{N}[a]$.

Proof. (a) Supposing $x \in \Omega_{N}(b, \beta),(3.2),(3.5)$, (3.6) imply

(3.7) $\left\|\pi_{N} x-\pi_{M} x\right\| \leq\left\|\pi_{N} x-x\right\|<\beta d\left(\pi_{N} x\right) \leq \operatorname{dist}\left(\pi_{N} x, \partial \Omega\right) / 2, \quad$ hence

$$
\begin{aligned}
\operatorname{dist}\left(\pi_{M} x, X \backslash \Omega\right) & \geq \operatorname{dist}\left(\pi_{N} x, \partial \Omega\right)-\left\|\pi_{N} x-\pi_{M} x\right\| \\
& >\operatorname{dist}\left(\pi_{N} x, \partial \Omega\right) / 2 \geq a, \\
\left\|\pi_{M} x\right\| & \leq\left\|\pi_{N} x-\pi_{M} x\right\|+\left\|\pi_{N} x\right\|<1+1 / b \leq 1 / a .
\end{aligned}
$$

Thus $\pi_{M} x \in \Omega[a]$. Since $\beta<1 / 2,(3.7)$ implies $d\left(\pi_{N} x\right) \leq 2 d\left(\pi_{M} x\right)$, therefore by $(3.2)$

$$
\left\|x-\pi_{M} x\right\| \leq\left\|x-\pi_{N} x\right\|<\beta d\left(\pi_{N} x\right) \leq \alpha d\left(\pi_{M} x\right),
$$

i.e. $x \in \Omega_{M}(a, \alpha)$.

(b) Choose $\beta<\min \{c, \gamma / 2\}$. Since $\lim _{n \rightarrow \infty} \pi_{n} x=x$, (3.5) implies $x \in$ $\Omega_{n}(2 c, \beta)$ for some $n$. Thus $\Omega_{n}(2 c, \beta)$ is a neighborhood of $x$ that is contained in $\Omega_{N}(c, \gamma)$ if $N \geq n$, by $\operatorname{part}(\mathrm{a})$.

(c) If $x=y+t e_{N+1} \in L_{N}[a]$ with $y=\pi_{N} x$ then $|t|<d^{N+1}(x)$, which by the definition of $d^{N+1}$ implies $\pi_{N} x=x-t e_{N+1} \in \Omega$. 
(d) If $x \in \Omega_{N}(c, \gamma)$, write $\pi_{N+1} x=\pi_{N} x+t e_{N+1}$. (3.7), (3.8) hold with $b, \beta, M$ replaced by $c, \gamma, N+1$. (3.8) implies $\pi_{N+1} x \in \Omega[a]$, and (3.7) implies $\left\|t e_{N+1}\right\|<\gamma d\left(\pi_{N} x\right)$. Hence if $|u| \leq 2|t|$, we have $\left\|u e_{N+1}\right\|<$ $\operatorname{dist}\left(\pi_{N} x, \partial \Omega\right) / 2$ by (3.6). Therefore

$\left\|\pi_{N+1} x+u e_{N+1}-\pi_{N} x\right\|<\left\|\pi_{N+1} x-\pi_{N} x\right\|+\operatorname{dist}\left(\pi_{N} x, \partial \Omega\right) / 2<\operatorname{dist}\left(\pi_{N} x, \partial \Omega\right)$,

and so $\pi_{N+1} x+u e_{N+1} \in \Omega$. This means $2|t| \leq d^{N+1}\left(\pi_{N+1} x\right)$, whence $\pi_{N+1} x \in L_{N}[a]$.

Proposition 3.5. Suppose $\Omega$ is pseudoconvex. Let $d$ be either $d_{0}$ or 1 , and in the latter case assume $\alpha<a$. If $c>2 a^{\prime} \geq 2 a$ and $4 \gamma<\alpha$, then any $u \in$ $\mathcal{O}\left(\Omega_{N}\left(a^{\prime}, \alpha\right) \cap \Omega_{N+1}(a, \alpha) ; F\right)$ can be approximated by $v \in \mathcal{O}\left(\Omega_{N+1}(a, \alpha) ; F\right)$, uniformly on $\Omega_{N}(c, \gamma)$.

Proof. By Proposition 3.4(a) $\Omega_{N}\left(a^{\prime}, \alpha\right) \cap \Omega_{N+1}(a, \alpha) \supset \Omega_{N}\left(2 a^{\prime}, \alpha / 2\right)$. Furthermore, this latter set is of type (B) for the splitting $X=\pi_{N} X \oplus \operatorname{Ker} \pi_{N}$ with base $\Omega\left[2 a^{\prime}\right] \cap \pi_{N} X$. Similarly, $\bar{\Omega}_{N}(c, \gamma) \subset \Omega_{N}\left(2 a^{\prime}, \alpha / 2\right)$ is also of type (B), with base $\bar{\Omega}_{N}[c] \cap \pi_{N} X$. This latter base is compact, and $\operatorname{psh}\left(\Omega \cap \pi_{N} X\right)$ convex by Proposition 3.3 ; hence it is $\mathcal{O}\left(\Omega \cap \pi_{N} X\right)$ convex by [H, Theorem 4.3.4]. Therefore by Proposition $3.2 u \mid \Omega_{N}\left(2 a^{\prime}, \alpha / 2\right)$ can be approximated by $w \in \mathcal{O}\left(\pi_{N}^{-1} \Omega ; F\right)$, uniformly on $\Omega_{N}(c, \gamma)$. According to Proposition 3.4(c)

$$
\pi_{N}^{-1} \Omega=\pi_{N+1}^{-1} \pi_{N}^{-1} \Omega \supset \pi_{N+1}^{-1} L_{N}[a] .
$$

This latter is of type (B) for the splitting $X=\pi_{N+1} X \oplus \operatorname{Ker} \pi_{N+1}$, with base $L_{N}[a]$. Since by Proposition 3.3 and [H, Theorem 4.3.4] the base is $\mathcal{O}\left(\Omega \cap \pi_{N+1} X\right)$ convex, Proposition 3.2 implies we can approximate $w$ by $w^{\prime} \in$ $\mathcal{O}\left(\pi_{N+1}^{-1} \Omega ; F\right)$, uniformly on \|\| -bounded subsets of $\pi_{N+1}^{-1} L_{N}[a]$. To finish the proof, notice that by Proposition 3.4(d,a) $\Omega_{N}(c, \gamma)$ is a \| \|-bounded subset of $\pi_{N+1}^{-1} L_{N}[a]$ and $\Omega_{N}(c, \gamma) \subset \Omega_{N+1}(a, \alpha) \subset \pi_{N+1}^{-1} \Omega$, so that $v=$ $w^{\prime} \mid \Omega_{N+1}(a, \alpha)$ will do.

Proposition 3.6. Let $d \equiv 1$, assume $2 \alpha<2 a \leq c$ and $\tau, \gamma \leq \alpha / 5$. If $x \in \Omega_{N}(c, \gamma)$ then $B(x, \tau d(x)) \subset \Omega_{N}(a, \alpha)$.

Proof. Since $\left\|x-\pi_{N} x\right\| \leq d\left(\pi_{N} x\right) / 2$, we have $d(x) \leq 2 d\left(\pi_{N} x\right)$. If $y \in$ $B(x, \tau d(x))$ then by $(3.2),(3.6)$

$$
\left\|\pi_{N} x-\pi_{N} y\right\| \leq\|x-y\|<\tau d(x) \leq 2 \tau d\left(\pi_{N} x\right) \leq \operatorname{dist}\left(\pi_{N} x, \partial \Omega\right) / 2 \text {, hence }
$$

$\operatorname{dist}\left(\pi_{N} y, X \backslash \Omega\right) \geq \operatorname{dist}\left(\pi_{N} x, \partial \Omega\right)-\left\|\pi_{N} x-\pi_{N} y\right\|>\operatorname{dist}\left(\pi_{N} x, \partial \Omega\right) / 2>a$, 


$$
\left\|\pi_{N} y\right\| \leq\left\|\pi_{N} x-\pi_{N} y\right\|+\left\|\pi_{N} x\right\|<1+1 / c<1 / a .
$$

By the last two inequalities $\pi_{N} y \in \Omega[a]$. Furthermore

$\left\|y-\pi_{N} y\right\| \leq\|y-x\|+\left\|x-\pi_{N} x\right\|+\left\|\pi_{N} x-\pi_{N} y\right\|<(4 \tau+\gamma) d\left(\pi_{N} x\right) \leq \alpha d\left(\pi_{N} x\right)$,

so that $y \in \Omega_{N}(a, \alpha)$.

With $0<\mu<1$ set now

$$
\mathfrak{B}(\mu)=\mathfrak{B}_{d}(\mu)=\{B(x, \mu d(x)): B(x, \mu d(x)) \subset \Omega\} .
$$

Proposition 3.7. Let $d \equiv 1$ and $4 \beta<4 b \leq c$, assume $12 \lambda \leq \mu$ and $10 \gamma, 50 \lambda \leq \beta$. Let $\sigma=\left(B\left(x_{j}, \mu d\left(x_{j}\right)\right)\right)_{j=0, \ldots, s} \subset \mathfrak{B}(\mu)$ and $\sigma^{\prime}=$ $\left(B\left(x_{j}, \lambda d\left(x_{j}\right)\right)\right)_{j=0, \ldots, s} \subset \mathfrak{B}(\lambda)$ be s-simplices, $s \geq 0$. Then any $u \in$ $\mathcal{O}\left(|\sigma| \cap \Omega_{N}(b, \beta) \cap \Omega_{N+1}(b, \beta) ; F\right)$ can be approximated by $v \in \mathcal{O}(X ; F)$, uniformly on $\left|\sigma^{\prime}\right| \cap \Omega_{N}(c, \gamma)$.

Proof. If $\left|\sigma^{\prime}\right| \cap \Omega_{N}(c, \gamma)=\emptyset$ there is nothing to prove. Otherwise let $x \in$ $\left|\sigma^{\prime}\right| \cap \Omega_{N}(c, \gamma)$. Since $\left\|x-x_{j}\right\|<\lambda d\left(x_{j}\right)<d\left(x_{j}\right) / 2$, we have

$$
d\left(x_{j}\right) / 2 \leq d(x) \leq 2 d\left(x_{j}\right) .
$$

We first claim $u$ is holomorphic on $B(x, 5 \lambda d(x))$. Indeed, on the one hand

$$
B(x, 5 \lambda d(x)) \subset \Omega_{N}(2 b, \beta / 2) \subset \Omega_{N}(b, \beta) \cap \Omega_{N+1}(b, \beta)
$$

by Propositions 3.6, 3.4(a); on the other $B(x, 5 \lambda d(x)) \subset|\sigma|$, since by (3.10)

$$
B(x, 5 \lambda d(x)) \subset B\left(x, 10 \lambda d\left(x_{j}\right)\right) \subset B\left(x_{j}, \mu d\left(x_{j}\right)\right) .
$$

We can apply Proposition 3.2 with $Y=X, L=D^{\prime}=D$ a singleton, to conclude that $u$ can be approximated by $v \in \mathcal{O}(X ; F)$, uniformly on $B(x, 4 \lambda d(x))$. Since

$$
\left|\sigma^{\prime}\right| \subset B\left(x_{0}, \lambda d\left(x_{0}\right)\right) \subset B\left(x, 2 \lambda d\left(x_{0}\right)\right) \subset B(x, 4 \lambda d(x)),
$$

the approximation is uniform on $\left|\sigma^{\prime}\right| \cap \Omega_{N}(c, \gamma)$.

Similarly one proves

Proposition 3.8. Let $d=d_{0}, 2 a \leq c<1$, and $18 \lambda \leq \mu<$ 1. Given s-simplices $\sigma=\left(B\left(x_{j}, \mu d\left(x_{j}\right)\right)\right)_{j=0, \ldots, s} \subset \mathfrak{B}(\mu)$ and $\sigma^{\prime}=$ $\left(B\left(x_{j}, \lambda d\left(x_{j}\right)\right)\right)_{j=0, \ldots, s} \subset \mathfrak{B}(\lambda), s \geq 0$, any $u \in \mathcal{O}(|\sigma| \cap \Omega[a] ; F)$ can be approximated by $v \in \mathcal{O}(X ; F)$, uniformly on $\left|\sigma^{\prime}\right| \cap \Omega[c]$. 


\section{Cohomology of special coverings.}

We shall use notation introduced in section 3. Again we let $X$ be as in (1.1), with $\log p(\theta, n)$ convex and even in $\theta$. We consider coverings $\mathfrak{B}_{d_{0}}(\mu)$ of $\Omega$ defined in (3.9), and we shall eventually prove

Proposition 4.1. Given $q=1,2, \ldots$, suppose $H^{s}\left(\Omega^{\prime}, \mathcal{F}\right)=0$ for any $1 \leq$ $s<q$ and pseudoconvex open $\Omega^{\prime} \subset X$. If $\Omega \subset X$ is pseudoconvex then $H^{q}\left(\mathfrak{B}_{d_{0}}(1 / 2), \mathcal{F}\right)=0$.

We shall repeatedly use the following special case of Proposition 2.1:

Proposition 4.2. Given $q=1,2, \ldots$, suppose $H^{s}\left(\Omega^{\prime}, \mathcal{F}\right)=0$ for any $1 \leq$ $s<q$ and pseudoconvex open $\Omega^{\prime} \subset X$. Let $\mathfrak{W}$ be a collection of pseudoconvex open $W \subset X$, and $\mathfrak{W}^{\prime}$ a refinement of $\mathfrak{W}, \bigcup \mathfrak{W}=\bigcup \mathfrak{W}^{\prime}$. Then the natural maps

$$
H^{q}(\mathfrak{W}, \mathcal{F}) \rightarrow H^{q}(\bigcup \mathfrak{W}, \mathcal{F}), \quad H^{q}(\mathfrak{W}, \mathcal{F}) \rightarrow H^{q}\left(\mathfrak{W}^{\prime}, \mathcal{F}\right)
$$

are monomorphisms.

To prove Proposition 4.1 we first introduce

$$
\mathfrak{C}(a)=\{B(x, a / 2): x \in \Omega[a]\} \subset \mathfrak{B}_{1}(a / 2), \quad 0<a<1,
$$

and study the groups $H^{q}(\mathfrak{C}(a), \mathcal{F})$.

Proposition 4.3. With the assumptions of Proposition 4.1, if $d \equiv 1$ and $2 \alpha<a<1 / 2$ then $\mathfrak{C}(a)$ covers $\Omega_{N}(2 a, \alpha)$, and $H^{q}(\mathfrak{C}(a), \mathcal{F}) \mid \Omega_{N}(2 a, \alpha)=0$.

Proof. We shall use notation as in Proposition 2.3 and in the lines preceding it. Writing $D(b)=\Omega[b] \cap \pi_{N} X, 0<b<1$, and $G=\left\{x \in \operatorname{Ker} \pi_{N}:\|x\|<\alpha\right\}$, we have $\Omega_{N}(b, \alpha)=D(b) \times G$. Fix $b \in(a, 2 a)$ and set $\omega=\Omega_{N}(2 a, \alpha)$, $\omega^{\star}=\Omega_{N}(b, \alpha)$. Each $\zeta \in \overline{D(b)}$ has a convex neighborhood $U^{\star} \subset \pi_{N} X$ such that $U^{\star}\left(\omega^{\star}\right)=U^{\star} \times G$ is contained in $B(\zeta, a / 2)$. Inscribe relatively compact convex open $U$ into each $U^{\star}$, and take a finite collection $\mathfrak{U}$ of $U$ 's that cover $\overline{D(a)}$, making sure the corresponding $U^{\star}$ 's cover $\overline{D(b)}$. Let

$$
\mathfrak{V}=\{D(a) \cap U: U \in \mathfrak{U}\}, \mathfrak{V}^{\star}=\left\{D(b) \cap U^{\star}: U \in \mathfrak{U}\right\} .
$$

Thus members of $\mathfrak{V}^{\star}, \mathfrak{V}^{\star}\left(\omega^{\star}\right)$ are pseudoconvex. Since each $V^{\star}\left(\omega^{\star}\right) \in$ $\mathfrak{V}^{\star}\left(\omega^{\star}\right)$ is contained in some $B(\zeta, a / 2) \in \mathfrak{C}(a), \mathfrak{C}(a)$ indeed covers $\left(\omega^{\star}\right.$ and $)$ 
$\omega=\Omega(2 a, \alpha)$. Further, there are refinement homomorphisms

$$
\begin{aligned}
& \rho^{1}: H^{q}\left(\mathfrak{C}(a) \mid \omega^{\star}, \mathcal{F}\right) \longrightarrow H^{q}\left(\mathfrak{V}^{\star}\left(\omega^{\star}\right), \mathcal{F}\right), \\
& \rho^{2}: H^{q}(\mathfrak{C}(a) \mid \omega, \mathcal{F}) \longrightarrow H^{q}\left(\mathfrak{V}^{\star}\left(\omega^{\star}\right) \mid \omega, \mathcal{F}\right),
\end{aligned}
$$

in fact monomorphisms by Proposition 4.2, for Proposition 3.1 implies balls $B(x, r)$ are convex. The restrictions $\rho_{1}, \rho_{2}$ of $\rho^{1}, \rho^{2}$ to $H^{q}(\mathfrak{C}(a), \mathcal{F}) \mid \omega^{\star}$ resp. $H^{q}(\mathfrak{C}(a), \mathcal{F}) \mid \omega$ are also monomorphisms, and can be included in a commutative diagram

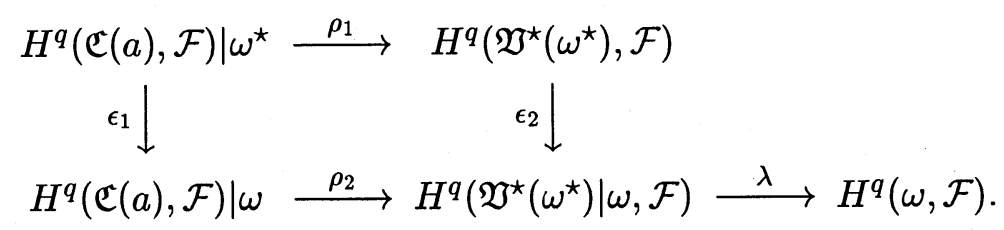

Here $\epsilon_{1}, \epsilon_{2}$ are induced by restriction, and $\lambda$ is the natural map from Čech to sheaf cohomology; again by Proposition $4.2 \lambda$ is a monomorphism. It is the content of Proposition 2.3 that $\lambda \epsilon_{2}=0$. Thus $\lambda \rho_{2} \epsilon_{1}=0$. Taking into account that $\lambda \rho_{2}$ is a monomorphism and $\epsilon_{1}$ an epimorphism, the proposition follows.

Proposition 4.4. With the assumptions of Proposition 4.1

$$
H^{q}(\mathfrak{C}(a), \mathcal{F}) \mid \Omega[16 a]=0 \quad \text { if } \quad 0<a<1 / 16 .
$$

Proof. We shall apply Lemma 2.2. Let $d \equiv 1, \alpha<a / 2, \Omega_{N}=\Omega_{N}(2 a, \alpha)$, $\omega_{N}=\Omega_{N}(8 a, \alpha / 10), \omega=\Omega[16 a], \mathfrak{U}=\mathfrak{C}(a) \subset \mathfrak{B}_{1}(a / 2)$,

$$
\mathfrak{U}^{\prime}=\{B(x, \alpha / 50): x \in \Omega[a]\} \subset \mathfrak{B}_{1}(\alpha / 50) .
$$

The assumptions of Lemma 2.2 are satisfied by Propositions $4.3\left(\Omega_{N} \subset\right.$ UU $), 3.4(\mathrm{a}, \mathrm{b}), 3.5$ (condition (ii) when $q=1$ ), 3.7 (condition (ii) when $q \geq 2$ ), 4.2, and 4.3. The conclusion of Lemma 2.2 is that the image of $H^{q}(\mathfrak{C}(a), \mathcal{F}) \mid \Omega[16 a]$ under the natural map

$$
H^{q}(\mathfrak{C}(a) \mid \Omega[16 a], \mathcal{F}) \rightarrow H^{q}(\Omega[16 a], \mathcal{F})
$$

is zero. Now Proposition 3.3 implies the members of $\mathfrak{C}(a) \mid \Omega[16 a]$ are pseudoconvex. Hence by Proposition 4.2, (4.1) is a monomorphism, whence $H^{q}(\mathfrak{C}(a), \mathcal{F}) \mid \Omega[16 a]=0$.

Proposition 4.5. With the assumptions of Proposition 4.1

$$
H^{q}\left(\mathfrak{B}_{d_{0}}(1 / 2), \mathcal{F}\right) \mid \Omega[b]=0 \quad \text { for any } \quad 0<b<1 .
$$


Proof. With $a=b / 16$ consider the map

$$
\mathfrak{C}(a) \ni B(x, a / 2) \mapsto B\left(x, d_{0}(x) / 2\right) \in \mathfrak{B}_{d_{0}}(1 / 2) .
$$

Since $B(x, a / 2) \subset B\left(x, d_{0}(x) / 2\right)$ if $x \in \Omega[a]$, this map induces a homomorphism of cochain complexes $C^{*}\left(\mathfrak{B}_{d_{0}}(1 / 2), \mathcal{F}\right) \rightarrow C^{*}(\mathfrak{C}(a), \mathcal{F})$, and the corresponding homomorphism in cohomology can be included in a commutative diagram

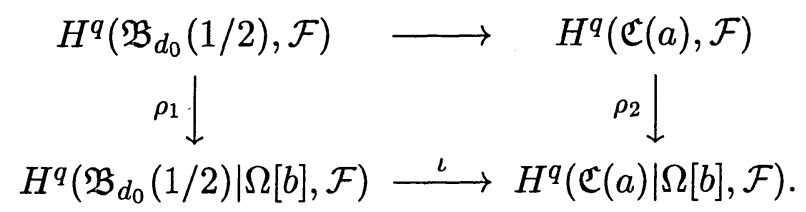

Here $\rho_{1}, \rho_{2}, \iota$ are the restriction resp. refinement homomorphisms. By Proposition $4.4 \rho_{2}=0$, hence $\iota \rho_{1}=0$. On the other hand $\iota$ is a monomorphism by Propositions 3.3, 4.2. Hence $\rho_{1}=0$, q.e.d.

Proof of Proposition 4.1. Again we shall apply Lemma 2.2. First suppose $q=$ 1. Let $d=d_{0}, \alpha<1 / 2$, put $\omega=\Omega, \Omega_{N}=\Omega_{N}(1 / N, \alpha), \omega_{N}=\Omega_{N}(4 / N, \alpha / 8)$, $N \geq 5\left(\right.$ and $\omega_{N}=\Omega_{N}=\emptyset$ if $\left.1 \leq N \leq 4\right), \mathfrak{U}=\mathfrak{U}^{\prime}=\mathfrak{B}_{d_{0}}(1 / 2)$. Then $\omega_{N} \subset \Omega_{N} \cap \Omega_{N+1}$ by Proposition 3.4(a). Condition (i) of Lemma 2.2 holds for if $x \in \Omega$ then $x \in \Omega[2 a]$ for some $a>0$ and Proposition 3.4(b) implies $x$ has a neighborhood that is contained in $\Omega_{N}(a, \alpha) \subset \Omega_{N}(4 / N, \alpha)$ for all but finitely many $N \geq 4 / a$. Condition (ii) follows from Proposition 3.5. Finally (3.5) implies $\Omega_{N} \subset \Omega[1 /(2 N)]$, so that $H^{1}\left(\mathfrak{B}_{d_{0}}(1 / 2), \mathcal{F}\right) \mid \Omega_{N}=0$ by Proposition 4.5. Therefore with Lemma 2.2 we conclude the map $H^{1}\left(\mathfrak{B}_{d_{0}}(1 / 2), \mathcal{F}\right) \rightarrow$ $H^{1}(\Omega, \mathcal{F})$ is zero. Since this map is a monomorphism by Proposition 4.2, $H^{1}\left(\mathfrak{B}_{d_{0}}(1 / 2), \mathcal{F}\right)=0$ as claimed.

When $q \geq 2$, let $\Omega_{N}=\Omega[1 / N], \omega_{N}=\Omega[2 / N], \mathfrak{U}=\mathfrak{B}_{d_{0}}(1 / 2), \mathfrak{U}^{\prime}=$ $\mathfrak{B}_{d_{0}}(1 / 36)$. Clearly $\omega_{N} \subset \Omega_{N} \subset \Omega_{N+1}$, and condition (i) of Lemma 2.2 holds. Condition (ii) follows from Proposition 3.8, and condition (iii) from Proposition 3.3, 4.2, and our assumptions. Finally, $H^{q}\left(\mathfrak{B}_{d_{0}}(1 / 2), \mathcal{F}\right) \mid \Omega_{N}=0$ is the content of Proposition 4.5. As before, Lemma 2.2 and Proposition 4.2 therefore imply $H^{q}\left(\mathfrak{B}_{d_{0}}(1 / 2), \mathcal{F}\right)=0$.

\section{Proof of Theorem 1.1.}

Proposition 4.1 has the following simple consequence:

Proposition 5.1. Suppose $X$ is as in (1.1) with $\log p(\theta, n)$ convex and even in $\theta$. Given $q=1,2, \ldots$ assume $H^{s}\left(\Omega^{\prime}, \mathcal{F}\right)=0$ for all $1 \leq s<q$ and 
pseudoconvex open $\Omega^{\prime} \subset X$. Let $\Omega \subset X$ be pseudoconvex open, and $f \in$ $H^{q}(\Omega, \mathcal{F})$. If $f \mid B(x, r / 2)=0$ whenever $B(x, r) \subset \Omega$ then $f=0$.

Proof. With $\mathfrak{B}_{d_{0}}(1 / 2)$ as in (3.9) we have $f \mid B=0$ for $B \in \mathfrak{B}_{d_{0}}(1 / 2)$ by assumption. Since $\mathfrak{B}_{d_{0}}(1 / 2)$ is Leray of order $q$, Proposition 2.1 implies $f$ is in the image of the map $H^{q}\left(\mathfrak{B}_{d_{0}}(1 / 2), \mathcal{F}\right) \rightarrow H^{q}(\Omega, \mathcal{F})$, whence $f=0$ by Proposition 4.1.

Proof of Theorem 1.1. We shall proceed by induction, and assume $H^{s}\left(\Omega^{\prime}, \mathcal{F}\right)=0$ is already known for all pseudoconvex open $\Omega^{\prime} \subset X$ and $1 \leq s<q$. At the price of replacing $p$ by $p^{\prime}(\theta, n)=\max \{p(\theta, n), p(-\theta, n)\}$ we can assume the weight $p(\theta, n)$ in (1.1) is even in $\theta$. Fix an arbitrary $f \in H^{q}(\Omega, \mathcal{F})$, let $\mathfrak{G}_{0}$ denote the collection of pseudoconvex open subsets of $X$, and $\mathfrak{G}$ the collection of those $G \in \mathfrak{G}_{0} \mid \Omega$ for which $f \mid G=0$. Let $\mathfrak{U} \subset \mathfrak{G}_{0}$ be a covering of $\Omega$ such that $f$ is in the image of the map $H^{q}(\mathfrak{U}, \mathcal{F}) \rightarrow H^{q}(\Omega, \mathcal{F})$; by Proposition $2.1 f \mid U=0$ if $U \in \mathfrak{U}$. Hence $\bigcup \mathfrak{G}=\Omega$. In the terminology of [L1, Lemma 8.2] $\mathfrak{G}$ is descending, and inductive by Proposition 5.1. The conclusion of that lemma is $\Omega=\bigcup \mathfrak{G} \in \mathfrak{G}$, i.e. $f=0$. Therefore $H^{q}(\Omega, \mathcal{F})=0$ as claimed.

\section{References.}

[B] G. E. Bredon, Sheaf Theory, 2nd ed., Springer, New York, 1997.

[D1] S. Dineen, Cousin's first problem on certain locally convex topological vector spaces, An. Acad. Brasil. Cienc. 48 (1976), 11-12.

[D2] Complex Analysis in Locally Convex Spaces, North Holland, Amsterdam, 1981.

[Do] A. Douady, Le problème des modules pour les sous espaces analytiques compacts d'un espace analytique donné, Ann. Inst. Fourier Grenoble, 16 (1966), 1-95.

[H] L. Hörmander, An Introduction to Complex Analysis in Several Variables, 3rd edition, North Holland, Amsterdam, 1990.

[L1] L. Lempert, The Dolbeault complex in infinite dimensions III. Sheaf cohomology in Banach spaces, Invent. Math. 142 (2000), 579-603.

[L2] Holomorphic approximation in Fréchet spaces, Commun. in Anal. and Geom. 11 (2003), 1-15. 
[Ma] P. Mazet, Analytic Sets in Locally Convex Spaces, North Holland, Amsterdam, 1984.

[MV] R. Meise and D. Vogt, Counterexamples in holomorphic functions on nuclear Fréchet spaces, Math. Z. 182 (1983), 167-177.

[N] P. Noverraz, Pseudo-convexité, convexité polynomiale et domaines d'holomorphie en dimension infinie, North Holland, Amsterdam, 1973.

Department of Mathematics

Purdue University, West Lafayette, IN 47907

USA

E-mail address: lempert@math.purdue.edu

ReCeived November 16, 2000. 